psychological distress and mental tiredness. In this study, we compared the results of the pretest-post test evaluation of participants in two groups of lupus patients with and without intervention. 24 lupus patients participated in this study. Then they divided randomly into two groups including patients with ACT intervention and patients without intervention. Both groups were matched for age, major organ involvement, education and economic level. At the beginning and end of the study, all patients filled out 3 questionnaires including Back hopelessness scale, Kessler psychological Distress Scale, and Krupp the fatigue severity score. After 8 consecutive secession of ACT therapy in cases, both groups were compared in scores of aforementioned questionnaires before and after therapy. In case group, hopeless, psychological distress, and fatigue severity were improved significantly compared with controls $(\mathrm{p}<0.01)$. Few studies on this peruse have been conducted until recently. Acceptance and commitment therapy is a method in which patient learns to accept negative thoughts as a challenge not a believe. They learn to try to accept their limitations instead of fighting them. It is a method of psychological flexibility and motivation to find the best ways to overcome those limitations instead of denying them. This study demonstrated that ACT psychotherapy reduces frustration, psychological distress and mental tiredness in lupus patients.

\section{PS6:113 LONG-TERM HYDROXYCHLOROQUINE USE: ARE LUPUS PATIENTS UNDERMONITORED?: A UNIVERSITY TEACHING HOSPITAL EXPERIENCE}

K Sunmboye, S Shaffu, M McCartney. Rheumatology department University hospitals of Leicester, Leicester, UK

\subsection{6/lupus-2018-abstract.157}

Hydroxychloroquine is an integral part of maintenance therapy in lupus patients. Recent UK guidelines recommend that patients on hydroxychloroquine need to have regular monitoring. The risk of retinal toxicity becomes significant after a cumulative dose of 1 gram or after 7 years of drug use. Monitoring with use of spectral domain optical coherence tomography (OCT) is required after 5 years of use from recent guidelines.

The University hospitals of Leicester which serves 1 million population has a diverse group of caucasian and black with minority ethnic (BME) groups. There are 293 patients with lupus on the rheumatology databse of the tertiary hospital. $194(66 \%)$ of them are on hydroxychloroquine. We performed an audit of patients that have regular eye monitoring whilst on hydroxychloroquine. Letters were sent out to the patients with lupus on hydroxychloroquine. 106 responses were obtained with a response rate of 55\%. 96 (91\%) were female and the remainder were male. The average age of the respondents was 49 years. The average duartion of hydroxychloroquine use was 79 months. 53\% of the respondents had been on Hydroxychloroquine for more than 5 years. $42 \%$ of all respondents were made of patients in the BME group. Of the 106 responders, only $86 \%$ (91) were aware that eye checks were required. Majority of the patients (60\%) who were not aware of the need for regular eye monitoring were in the BME group. $71 \%$ of patients who had not had regular eye monitoring had other co-morbidities other than lupus. 1 in 5 of patients who did not have regular eye monitoring had other DMARDs alongside hydroxychloroquine. 20\% of this patient cohort were not compliant with regular eye monitoring after use of hydroxychloroquine after 5 years or more.

Ethnicity, disease factors, use of other DMARDs and duration of hydroxychloroquine use may play a role in determing patient compliance with regular eye monitoring. We recommended that more should be done to continually educate patients about the need to have yearly eye checks.

\section{PS6:114 HOW TO DIAGNOSE LUPUS ENTERITIS EARLY? LESSONS LEARNED FROM A MULTICENTER CASE SERIES}

${ }^{1} \mathrm{M}$ Luís, ${ }^{1} \mathrm{~L}$ Brites, ${ }^{2} \mathrm{AC}$ Duarte, ${ }^{3} \mathrm{~V}$ Teixeira, ${ }^{3} \mathrm{C}$ Macieira, ${ }^{2} \mathrm{MJ}$ Santos, ${ }^{1} \mathrm{~L}$ Inês. ${ }^{1} \mathrm{C}$ entro Hospitalar e Universitário de Coimbra - Department of Rheumatology, Coimbra, Portugal; ${ }^{2}$ Hospital Garcia de Orta - Department of Rheumatology, Lisboa, Portugal; ${ }^{3}$ Centro Hospitalar Lisboa Norte - Department of Rheumatology, Lisboa, Portugal

\subsection{6/lupus-2018-abstract.158}

Introduction Lupus enteritis (LE) is a rare, potentially lifethreatening manifestation of systemic lupus erythematosus (SLE). Early diagnosis is crucial for early treatment and prevention of serious complications such as ischaemic enteritis, bowel infarction with bleeding and/or perforation and peritonitis. The objective of this case review is to identify strategies for early diagnosis of LE.

Methods Retrospective analysis of patients with SLE (fulfilling ACR 1997 and/or SLICC classification criteria) and presenting LE from three tertiary SLE centres was conducted. The diagnosis was based on clinical and imaging features consistent with LE and exclusion of other causes of GI disorders.

Results We report six cases of LE (female: 100\%; age range: 16-55 years). All presented with acute onset abdominal pain, nausea and vomiting at the emergency department. One patient had lupus enteritis as inaugural manifestation of SLE. Of the remaining five, one presented at the previous visit to the lupus clinic with clinically active disease and two had serologically active/clinically inactive SLE. High anti-dsDNA antibodies and low serum complement were universally present at time of the LE event. Abdominal ultrasound was the first imaging exam to be performed in the emergency unit. In all cases it showed bowel wall thickening, dilatation of intestinal segments, increased reflectivity of mesenteric fat and mild ascites, raising the suspicion of LE and immediate start of treatment. These features were later confirmed by CT scan.

Discussion Abdominal ultrasound can be a reliable first line diagnostic tool for LE. It is a handy and radiation-free technique, an important advantage in women of child-bearing age.

\section{PS6:115 CYCLOPHOSPHAMIDE AS INDUCTION TREATMENT OF LUPUS NEPHRITIS IN A GROUP OF TUNISIAN PATIENTS: MONTHLY HIGH-DOSE OR BIWEEKLY LOW- DOSE PULSES?}

T Ben Salem, M Tougorti, M Lamloum, I Ben Ghorbel, MH Houman. Department of Internal Medicine, La Rabta University Hospital, Tunis, Tunisia

\subsection{6/lupus-2018-abstract.159}

Background Cyclophosphamide is an effective treatment of lupus nephritis (LN) but have many side effects especially with high doses. Two regimens are used: monthly high dose pulses or biweekly low dose pulses (Eurolupus protocol). LN 
prognosis is different according to ethnicity. There are no controlled trials in African populations.

Objectives Evaluate the efficiency of two regimens of cyclophosphamide in the treatment of LN.

Patients and methods 48 patients with histological proven LN and treated with cyclophosphamide were included. Patients were divided in 2 groups: Group 1 (6 monthly high-dose intravenous cyclophosphamide) and Group 2 (Eurolupus protocol). We evaluate complete remission rate (absence of proteinuria and renal failure), partial remission (decrease of proteinuria more than $50 \%$ without renal failure) and renal relapses into the 2 groups.

Results There were 32 patients in group 1 and 16 patients in group 2. Mean age at time of LN diagnosis was 30 years in group 1 and 28.3 years in group 2. Mean level of proteinuria was $3 \mathrm{~g} /$ day (group 1) and $3.73 \mathrm{~g} /$ day (group 2). Hematuria was found in 16 and 4 patients respectively. Five patients in group 1 and three in group 2 were in renal failure at time of LN flare. Lupus nephritis was class III in 10 (group 1) and 4 cases (group 2), class IV (group 1: $n=14$, group 2: $n=9$ ), class III/V (group $1: n=5$, group $2: \mathrm{n}=2$ ) and class IV/V (3 in group 1). Complete remission was achieved in $62.5 \%$ of case in group $1 \%$ and $56.3 \%$ patients in group $2(\mathrm{p}=0.6)$. Partial remission was obtained in $25 \%$ of cases in both groups. Renal relapses occurred in 12.5\% (group1) and $18.8 \%$ (group 2) of patients $(\mathrm{p}=0.56)$. None had end stage renal failure. The average duration of follow up was of 5.32 years in the group 1 and 5.49 years in group 2 .

Conclusion Monthly cyclophosphamide pulses regimen was similar to Eurolupus protocol in our group. These results encourage us to use biweekly cyclophosphamide pulses which are safer in SLE patients. More studies are needed to confirm these results.

\section{PS6:116 DIET HABITS IN PATIENTS WITH SYSTEMIC LUPUS ERYTHEMATOSUS}

${ }^{1} \mathrm{M}$ Petric, ${ }^{1,2} \mathrm{M}$ Radic, ${ }^{1,2} \mathrm{D}$ Perkovic, ${ }^{2} \mathrm{~J}$ Bozic, ${ }^{1} \mathrm{M}$ Petric, ${ }^{1,2} \mathrm{D}$ Marasovic Krstulovic, ${ }^{1} \mathrm{~K}$ Boric, ${ }^{1}$ I Bozic, ${ }^{1}$ I Erceg, ${ }^{1,2}$ D Martinovic Kaliterna. ${ }^{1}$ University Hospital of Split, Croatia; ${ }^{2}$ University of Split School of Medicine, Split, Croatia

\subsection{6/lupus-2018-abstract. 160}

Objective Many patients with systemic lupus erythematosus (SLE) are interested in diet advices. We wanted to investigate which diet habits are most common in our patients, and which of them are in correlation with laboratory parameters that are related to disease remission, such as normal values of serum complement and 24 hour proteinuria.

Methods We included 76 patient with SLE in remission, in age between 21 and 75. They fulfilled 23-item questionnaire about weekly diet habits. Basic anthropometric data, disease duration, levels of C3 and C4 complement components and 24 hour proteinuria were recorded and analysed in correlation with diet habits.

Results Majority of our patients had normal BMI (between 18.5 and $25 \mathrm{~kg} / \mathrm{m} 2$ ), prefered to eat healthy food and did regular weekly workout. Milk, meat, fruits, vegetables, pasta, rice and bread were the most abundant food. Lower serum values of C3 were found in $34(44.7 \%)$ cases, and lower values of C4 were found in $28(36.8 \%)$ cases. Only 5 (6.6\%) patients had significant 24 hour proteinuria higher than $3.5 \mathrm{~g}$ and another 7 (9.2\%) had proteinuria higher than $1 \mathrm{~g}$. Lower values of C3 were found in patients who often consumed meat $(p=0.015)$ and fast food $(p=0.060)$, and those patients who more often consumed fast food or fried food had lower levels of C4 ( $p=0.043$ and $p=0.051)$.

Conclusions There is an evidence that food rich in proteins and calories can lower serum complement levels. As clinicians, we should always advice our SLE patients to eat a lot of fish, fruits and fresh vegetables, although there is no strong support for that. More studies with dietary interventions have to be done before final recommendations can be made.

\section{PS6:118 OBJECTIVE MEASUREMENTS OF SLEEP DISORDERS AND PSYCHIATRIC COMORBIDITIES IN A COHORT OF PATIENTS WITH SYSTEMIC LUPUS ERYTHEMATOSUS}

${ }^{1,2} \mathrm{U}$ Faraguna, ${ }^{3} \mathrm{C}$ Porciani, ${ }^{4} \mathrm{R}$ Vagelli, ${ }^{2} \mathrm{M}$ di Galante, ${ }^{3,4} \mathrm{C}$ Tani, ${ }^{4} \mathrm{C}$ Stagnaro, ${ }^{3,4} \mathrm{M}$ Mosca. ${ }^{1}$ Dept. of Translational Reserach - University of Pisa, Italy; ${ }^{2}$ IRCCS Stella Maris, Calambrone, ITALY; ${ }^{3}$ Dept. of Clinical and Experimental Medicine, Pisa, Italy; ${ }^{4}$ AOUP -Rheumatology Unit, Pisa, Italy

\subsection{6/lupus-2018-abstract.161}

Background Depression is one of the most frequent disorders in SLE, from $17 \%$ to $75 \%$ of prevalence, although subtle neuropsychiatric syndromes like symptoms of depressive and anxiety axes are often considered as 'non-neuropsychiatric SLE'. Recent data suggest SLE patients also suffer from sleep disturbances like frequent awakenings and unrestorative sleep, and worse sleep quality has been found to be a fellow traveller with this disease.

Aims to objectively evaluate sleep in SLE patients in comparison with a cohort of age and sex-matched controls and to find possible relationships with disease manifestations and to find the factors that have greater impact on mood disorders in SLE group.

Methods sleep was evaluated in 41 SLE patients and 36 controls using actigraphy. The presence of mood disorders, temperament, health-related quality of life and perception of sleep were evaluated with specific questionnaires: Beck Depression Inventory (BDI), Self Rating Anxiety Scale (SAS), Brief COPE, Pittsburgh Sleep Quality Index (PSQI), Insomnia Severity Index (ISI), Perceived Stress Scale (PSS), Resilience Scale for adult, Functional Assessment of Chronic Illness Therapy (FACIT) Fatigue Scale, Brief TEMPS-M, Lupus QoL and Short Form Health survey 36 (in SLE patients and controls respectively).

Results The strongest predictors of the SLE group were higher scores in BDI and SAS index, lower Sleep Efficiency and greater Total Sleep Time.

Statistically significant differences were found between depressed SLE patients and non-depressed SLE patients in several parameters. Lower scores in FACIT fatigue scale, burden to others, pain and body image domains and higher PSS score were found in depressed SLE patients. In SLE group, FACIT score was strongly negative correlated with BDI score and positively correlated with physical domain.

Fibromyalgic SLE patients had lower scores in pain domain when compared with non-fibromyalgic SLE patients. Instead, no difference in pain domain was found between patients with joint involvement and patients without joint involvement, addressing fibromyalgia as the factor with greater impact over pain.

Conclusion SLE is a chronic disease that has great impact on mood and sleep quality and identification of this problems and consequent therapeutic interventions may improve the quality of life of these patients. 